\title{
Comparison of clinical features and perinatal outcomes between pre-variant and post-variant periods in pregnant women with SARS-CoV-2: analysis of 1935 cases
}

\author{
Dilek Sahin ${ }^{1} \cdot$ Atakan Tanacan $^{2}$ D $\cdot$ Ali Taner Anuk ${ }^{2} \cdot$ Selcan Sinaci ${ }^{2} \cdot$ Berhan Besimoglu $^{2} \cdot$ Deniz Oluklu $^{2}$. \\ Derya Uyan Hendem ${ }^{2}$. Dilek Menekse Beser ${ }^{2}$. Muradiye Yildirim² ${ }^{2}$ Bedri Sakcak ${ }^{2}$. Seyit Ahmet Erol ${ }^{2}$. \\ Yeliz Colakoglu ${ }^{2}$. Sule Goncu Ayhan ${ }^{2} \cdot$ Ezgi Turgut $^{2} \cdot$ Serpil Unlu $^{3} \cdot$ Fuat Emre Canpolat $^{4} \cdot$ Seval Izdes $^{5} \cdot$ Sema Turan $^{6}$. \\ Aziz Ahmet Surel ${ }^{7}$. Ozlem Moraloglu Tekin ${ }^{1}$
}

Received: 21 January 2022 / Accepted: 25 February 2022 / Published online: 7 March 2022

(c) The Author(s), under exclusive licence to Springer-Verlag GmbH Germany, part of Springer Nature 2022

\begin{abstract}
Purpose To compare the clinical features and perinatal outcomes of pregnant women with severe acute respiratory syndrome coronavirus 2 (SARS-CoV-2) in the pre-variant and post-variant periods.

Methods This prospective cohort study includes pregnant women with SARS-CoV-2 who were followed-up at Ankara City Hospital between 11, March 2020 and 15, September 2021. Demographic features, clinical characteristics and pregnancy outcomes were compared between the pre-variant $(n=1416)$ and post-variant $(n=519)$ groups.

Results The rates of severe and critical cases significantly increased in the post-variant group $(9.7 \%$ vs $2 \%, p<0.001)$. The rates of respiratory support $(26.8 \%$ vs $7.3 \%, p<0.001)$, ICU admission $(12.9 \%$ vs $1.8 \%, p<0.001)$ and maternal mortality $(2.9 \%$ vs $0.4 \%, p<0.001)$ were significantly higher in the post-variant group. A significant increase was observed for pregnancy complications in the post-variant group $(45.6 \%$ vs $18.8 \%, p=0.007)$. The rates of preterm delivery $(26.4 \%$ vs $4.4 \%$, $p<0.001$ ) and NICU admission ( $34 \%$ vs $18.8 \%, p<0.001$ ) were significantly higher in the post-variant group. Positive, weak, statistically significant correlations were observed between the post-variant period, disease severity and maternal mortality $(r=0.19, r=0.12$ and $p<0.001)$.
\end{abstract}

Conclusion Post-variant COVID-19 period was associated with a severe course of the disease and increased rates of adverse obstetric outcomes in pregnant patients.

Keywords COVID-19 $\cdot$ SARS-CoV-2 $\cdot$ Pregnancy $\cdot$ Obstetric complications $\cdot$ Disease severity $\cdot$ COVID-19 variants

Atakan Tanacan

atakantanacan@yahoo.com

1 Department of Obstetrics and Gynecology, Turkish Ministry of Health Ankara City Hospital, University of Health Sciences, Ankara, Turkey

2 Department of Obstetrics and Gynecology, Turkish Ministry of Health Ankara City Hospital, 06800 Ankara, Turkey

3 Department of Infectious Diseases, Turkish Ministry of Health Ankara City Hospital, Ankara, Turkey

4 Division of Neonatology, Department of Pediatrics, Head of Center for Clinical Research, Turkish Ministry of Health Ankara City Hospital, University of Health Sciences, Ankara, Turkey
5 Intensive Care Clinic, Turkish Ministry of Health Ankara City Hospital, University of Health Sciences, Ankara, Turkey

6 Intensive Care Clinic, Ankara Yildirim Beyazit University, Turkish Ministry of Health Ankara City Hospital, Ankara, Turkey

7 Coordinator Head Physician of Turkish Ministry of Health Ankara City Hospital, Ankara, Turkey 


\section{Introduction}

Coronavirus disease 2019 (COVID-19) caused by severe acute respiratory syndrome coronavirus 2 (SARS-CoV-2) has been in the center of the world's attention since the beginning of the pandemic. This novel viral disease not only caused significant mortality and morbidity but also resulted in radical changes in our daily lives [1,2]. Health care systems have been struggling to control the spread of COVID-19 and health care workers have been working under extremely tough conditions to heal infected individuals for approximately 2 years [3, 4]. Although significant progress has been achieved due to effective health policies, lifestyle changes, legal regulations, and widespread vaccination programs, COVID-19 has still not been fully controlled, especially due to the emergence of new variants $[5,6]$.

Pregnant women with COVID-19 have higher risks for severe disease, need for mechanical ventilation, intensive care unit (ICU) admission, and maternal death [7-11]. Moreover, higher rates of obstetric complications like preterm delivery, preterm premature rupture of membranes, and fetal distress are observed in pregnancies complicated by COVID-19 $[12,13]$. There are recent publications in the literature indicating a worse prognosis in pregnant women infected by new SARS-CoV-2 variants [14-22]. However, our knowledge is still limited on the impact of new SARS-CoV-2 variants on maternal COVID-19 prognosis and pregnancy outcomes. For this reason, more data are necessary to achieve more precise results.

This study aims to compare the clinical features and perinatal outcomes of pregnant women with SARS-CoV-2 in the pre-variant and post-variant periods.

\section{Materials and methods}

This prospective cohort study includes pregnant women with SARS-CoV-2 who were followed-up at the Department of Obstetrics and Gynecology, Ankara City Hospital between 11, March 2020 and 15, September 2021. All consecutive SARS-CoV-2-positive cases confirmed by realtime polymerase chain reaction (RT-PCR) on nasopharyngeal and oropharyngeal samples were evaluated. Written informed consent was obtained from all participants. The study protocol was approved by the Turkish Ministry of Health and the institutional ethics committee with reference number E2-21-294.

Ankara City Hospital is one of the leading pandemic centers in Turkey dealing with highly complicated COVID-19 patients. Sahin et al. regularly updated their experience on pregnant COVID-19 patients and shared their findings with the literature with three published studies [23-25]. The present study is the latest update of this group. The first variant case in Ankara City Hospital was detected on 20, February 2021. Due to cost-effective issues, variant classification is not a routine part of clinical practice in Turkey. However, the majority of variant cases evaluated for research purposes were the Delta variants. For this reason, the authors divided the patients into two groups based on the determined periods to assess the impact of COVID-19 variants on pregnant women: (1) pre-variant group, (2) post-variant group. Maternal age, previous obstetric history, prepregnancy body-mass-index (BMI), comorbid diseases, gestational age at diagnosis, pregnancy trimester at diagnosis, initial symptoms, close contact with a confirmed or suspected case, abnormal vital signs at admission to hospital, pregnancy-specific medications, COVID-19 therapy, disease severity, respiratory support, ICU admission, maternal mortality, hospitalization rate, length of hospital stay, initial hemoglobin $(\mathrm{Hg})$, hematocrit (Hct), leukocyte, neutrophil, lymphocyte, neutrophil-to-lymphocyte ratio (NLR), platelet, erythrocyte sedimentation rate (ESR), C-reactive protein (CRP), procalcitonin, interleukin 6 (IL-6), ferritin, blood urea nitrogen (BUN), creatinine, liver enzymes, lactate dehydrogenase (LDH), D-dimer, creatine kinase MB (CK-MB), troponin, hypokalemia rate, radiologic imaging findings, pregnancy complications, delivery status, time interval between diagnosis and delivery, route of delivery, cesarean indications, labor anesthesia, spontaneous labor rate, preterm delivery rate, gestational age at delivery, birth weight, 1-5 min Apgar scores, neonatal intensive care unit (NICU) admission, neonatal SARS-CoV-2 positivity and SARS-CoV-2 positivity in breastmilk were recorded for both groups. Demographic features, clinical characteristics, laboratory test results, radiologic imaging findings, obstetric and neonatal outcomes were compared between the groups. Furthermore, a correlation analysis was performed between disease severity, maternal mortality, and the post-variant period. All cases were managed according to the current scientific consensus by a multidisciplinary team and the severity of COVID-19 was assessed according to the national guideline [26-29]. Presence of any sign and symptom for COVID-19 without lower respiratory system involvement was defined as mild COVID-19. Evidence of lower respiratory disease by clinical assessment or imaging and a saturation of oxygen $(\mathrm{SaO} 2) \geq 94 \%$ on room air at sea level was defined as moderate COVID-19. Respiratory frequency $>30$ breaths per minute, $\mathrm{SaO} 2<94 \%$ on room air at sea level,ratio of arterial partial pressure of oxygen to fraction of inspired oxygen $(\mathrm{PaO} 2 / \mathrm{FiO} 2)<300$, or lung infiltrates $>50 \%$ was defined as severe COVID-19. Presence of respiratory failure, septic shock, and/or multiple 
organ dysfunction was defined as critical COVID-19. All cases were managed according to the Turkish Ministry of Health, General Directorate of Public Health, COVID-19 (SARS-CoV-2 infection) Guideline, Scientific Committee Report. The multidisciplinary team consisted of obstetricians, maternal-fetal medicine specialists, neonatologists, infectious disease specialists and radiologists [26-29].

Statistical analyses were performed using the Statistical Package for the Social Sciences (SPSS.22, IBM SPSS Statistics for Windows, Version 22.0 Armonk, NY: IBM Corp.). Descriptive analyses were presented as means and standard deviations for normally distributed values. Median and interquartile values were used for variables that were not normally distributed. Student's t test was used for comparing mean values between the groups. Mann-Whitney U test was performed to compare the median values between the groups. Categorical variables were presented by numbers and percentages. The chi-square test was used to compare categorical variables between the groups. Correlation analysis was performed by the Spearman test. A two-tailed $p$ value $<0.05$ was regarded as statistically significant.

\section{Results}

There were 1416 and 519 patients in the pre-variant and post-variant groups, respectively. None of the patients included in the study were vaccinated for COVID-19. A comparison of demographic features and clinical characteristics between the pre-variant and post-variant groups is summarized in Table 1. Comorbidities such as obesity was observed significantly more often in the pre-variant group ( $23.1 \%$ vs $17.1 \%, p=0.005)$. The rate of asymptomatic cases was significantly lower in the post-variant group $(40.1 \%$ vs $30.1 \%, p<0.001$ ). The rate of oxygen saturation $\leq 93 \%$ was significantly higher in the post-variant group. On the other hand, the rate of fever was significantly higher in the prevariant group. The rate of pregnancy-specific medications was significantly higher in the post-variant period due to an increase in the administration of antenatal corticosteroids $(p<0.05)$. Moreover, the rate of COVID-19 therapy was significantly higher in the post-variant group $(55.1 \%$ vs $68.4 \%, p<0.001)$.. The rates of severe and critical cases significantly increased in the post-variant group $(9.7 \%$ vs $2 \%, p<0.001)$. Moreover, the rates of respiratory support (26.8\% vs $7.3 \%, p<0.001)$, ICU admission $(12.9 \%$ vs $1.8 \%$, $p<0.001)$ and maternal mortality ( $2.9 \%$ vs $0.4 \%, p<0.001)$ were significantly higher in the post-variant group. However, the hospitalization rate was significantly lower in the postvariant group $(55.9 \%$ vs $23.3 \%, p<0.001)$.

Comparison of initial laboratory test results and radiologic imaging findings between the pre-variant and postvariant groups is shown in Table 2. Higher values for leukocyte, neutrophil, CRP, ferritin, liver enzymes, LDH, $\mathrm{D}$-dimer, and troponin were observed in the post-variant group. Lymphocytopenia and hypokalemia rates were also significantly higher in the post-variant group. Although the rates of radiologic imaging and suspicious radiologic findings consistent with COVID-19 were similar between the groups, a significant increase was observed for pulmonary computerized tomography angiography in the post-variant group $(p<0.05)$.

A comparison of obstetric and neonatal outcomes between the pre-variant and post-variant groups is shown in Table 3. A significant increase was observed for pregnancy complications in the post-variant group $(45.6 \%$ vs $18.8 \%, p=0.007$ ). Prominent increases were observed for miscarriage, cholestasis of pregnancy, gestational diabetes, preterm labor, and placental abruption $(p<0.05)$. The rates of cesarean section performed for worsening in maternal health condition and general anesthesia increased significantly in the post-variant group $(p<0.05)$. Furthermore, the rates of preterm delivery $(26.4 \%$ vs $4.4 \%, p<0.001)$ and NICU admission ( $34 \%$ vs $18.8 \%, p<0.001)$ were significantly higher in the post-variant group.

The correlation of the post-variant period with disease severity and maternal mortality is shown in Table 4 . Positive, weak, statistically significant correlations were observed between the post-variant period, disease severity, and maternal mortality ( $r=0.19$ for disease severity, $r=0.12$ for maternal mortality, and $p<0.001$ for both).

\section{Discussion}

Severe COVID-19, ICU admission, maternal mortality, and obstetric complication rates were significantly higher in the post-variant group compared to the pre-variant group in the present study. These results indicated a worse prognosis for pregnant women in the post-variant period. Although the experience of physicians dealing with pregnant women with COVID-19 has increased significantly since the beginning of the pandemic, COVID-19 still appears to be a major threat to the pregnant population.

Various mutations have been observed in the SARS$\mathrm{CoV}-2$ genome over time. Although the majority of them have no significant effect on the course of COVID-19, some variants have raised serious concern due to their rapid emergence. These variants are defined as variants of concern. Alpha (B.1.1.7 lineage), Beta (B.1.351 lineage), Delta (B.1.617.2 lineage), and Gamma (P.1 lineage) were the most common variants reported in the literature. These variants are more transmissible and they have the potential for a worse prognosis [30-32]. The impact of new SARS-CoV-2 variants on pregnant women has been investigated in recent studies [14-20,33]. A national population-based prospective 
Table 1 Comparison of demographic features and clinical characteristics between the pre-variant and post-variant groups

\begin{tabular}{|c|c|c|c|}
\hline Variables & Pre-variant group $(n=1416)$ & $\begin{array}{l}\text { Post-variant group } \\
(n=519)\end{array}$ & $p$ value \\
\hline Maternal age (years) $($ mean \pm SD) $(\min -\max )$ & $28.47 \pm 5.63(17-47)$ & $29.37 \pm 5.55(18-45)$ & 0.22 \\
\hline Advanced maternal age ( $\geq 35$ years) $(n, \%)$ & $219(15.4 \%)$ & $96(18.5 \%)$ & 0.11 \\
\hline Gravidity (median)( IQR, min-max) & $2(2,0-10)$ & $2(2,0-12)$ & 0.12 \\
\hline Parity (median) (IQR, min-max) & $1(2,0-7)$ & $1(2,0-9)$ & 0.76 \\
\hline Living child (median) (IQR, min-max) & $1(2,0-7)$ & $1(2,0-6)$ & 0.68 \\
\hline Previous miscarriage (median) (IQR, min-max) & $0(0,0-6)$ & $0(1,0-6)$ & 0.07 \\
\hline Prepregnancy BMI $\left(\mathrm{kg} / \mathrm{m}^{2}\right)(\operatorname{mean} \pm \mathrm{SD})(\min -\max )$ & $26.75 \pm 5.34(18-45)$ & $26.78 \pm 5.18(21-42)$ & 0.97 \\
\hline Comorbid disease $(n, \%)$ & $326(23.1 \%)$ & $89(17.1 \%)$ & 0.005 \\
\hline Obesity $(n, \%)$ & $169(11.9 \%)$ & $6(1.2 \%)$ & $<0.001$ \\
\hline Hypothyroidism $(n, \%)$ & $72(5.1 \%)$ & $37(7.1 \%)$ & 0.08 \\
\hline Hypertension $(n, \%)$ & $20(1.4 \%)$ & $12(2.3 \%)$ & 0.17 \\
\hline Asthma $(n, \%)$ & $19(1.3 \%)$ & $15(2.8 \%)$ & 0.02 \\
\hline Diabetes mellitus type $2(n, \%)$ & $12(8.5 \%)$ & $2(0.3 \%)$ & 0.28 \\
\hline Rheumatological disease $(n, \%)$ & $10(0.7 \%)$ & $4(0.7 \%)$ & 0.88 \\
\hline Cardiovascular disease $(n, \%)$ & $8(0.5 \%)$ & $2(0.3 \%)$ & 0.62 \\
\hline Diabetes mellitus type $1(n, \%)$ & $5(0.3 \%)$ & $2(0.3 \%)$ & 0.92 \\
\hline Epilepsy $(n, \%)$ & $4(0.3 \%)$ & $6(1.1 \%)$ & 0.017 \\
\hline Renal disease $(n, \%)$ & $3(0.2 \%)$ & $3(0.5 \%)$ & 0.19 \\
\hline $\operatorname{ITP}(n, \%)$ & $2(0.1 \%)$ & $0(0 \%)$ & 0.79 \\
\hline Thalessemia minor $(n, \%)$ & $2(0.1 \%)$ & $0(0 \%)$ & 0.79 \\
\hline Gestational age at diagnosis (weeks) $(\operatorname{mean} \pm \mathrm{SD})(\min -\max )$ & $25.59 \pm 11.13(4-41)$ & $26.36 \pm 9.65(5-40)$ & 0.12 \\
\hline \multicolumn{4}{|l|}{ Pregnancy trimester at diagnosis } \\
\hline First $(n, \%)$ & $311(22 \%)$ & $84(16.2 \%)$ & 0.001 \\
\hline Second $(n, \%)$ & $433(30.6 \%)$ & $161(31 \%)$ & \\
\hline Third $(n, \%)$ & $672(47.5 \%)$ & $274(52.8 \%)$ & \\
\hline \multicolumn{4}{|l|}{ Initial symptoms } \\
\hline Asymptomatic $(n, \%)$ & $567(40.1 \%)$ & $156(30.1 \%)$ & $<0.001$ \\
\hline Symptomatic $(n, \%)$ & $849(59.9 \%)$ & $363(69.9 \%)$ & \\
\hline Cough $(n, \%)$ & $392(27.7 \%)$ & $180(34.7 \%)$ & 0.04 \\
\hline Myalgia $(n, \%)$ & $350(24.7 \%)$ & $140(27 \%)$ & 0.86 \\
\hline Dyspnea $(n, \%)$ & $233(16.4 \%)$ & $155(29.9 \%)$ & $<0.001$ \\
\hline Headache $(n, \%)$ & $127(8.9 \%)$ & $38(7.1 \%)$ & 0.07 \\
\hline Anosmia $(n, \%)$ & $109(7.7 \%)$ & $15(2.9 \%)$ & $<0.001$ \\
\hline Sore throat $(n, \%)$ & $103(7.3 \%)$ & $40(7.7 \%)$ & 0.91 \\
\hline Nausea-vomiting $(n, \%)$ & $103(7.3 \%)$ & $45(8.7 \%)$ & 0.58 \\
\hline Ageusia $(n, \%)$ & $86(6.1 \%)$ & $14(2.7 \%)$ & 0.001 \\
\hline Fever $(n, \%)$ & $83(5.8 \%)$ & $45(8.7 \%)$ & $<0.001$ \\
\hline Nasal congestion $(n, \%)$ & $79(5.6 \%)$ & $17(3.3 \%)$ & 0.015 \\
\hline Diarrhea $(n, \%)$ & $53(3.7 \%)$ & $17(3.3 \%)$ & 0.41 \\
\hline Chest pain $(n, \%)$ & $31(2.2 \%)$ & $22(4.2 \%)$ & 0.03 \\
\hline Close contact with a confirmed or suspected case $(n, \%)$ & $188(13.3 \%)$ & $61(11.8 \%)$ & 0.36 \\
\hline \multicolumn{4}{|l|}{ Abnormal vital signs at admission to hospital } \\
\hline Tachycardia (heart rate $\geq 100 / \min )(n, \%)$ & $327(23.1 \%)$ & $151(29.1 \%)$ & 0.007 \\
\hline Fever (body temperature $\left.\geq 38^{\circ} \mathrm{C}\right)(n, \%)$ & $83(13.3 \%)$ & $45(8.7 \%)$ & $<0.001$ \\
\hline Tachypnea (respiratory rate $\geq 20 / \min )(n, \%)$ & $45(3.2 \%)$ & $42(8.1 \%)$ & $<0.001$ \\
\hline Oxygen saturation $\leq 93 \%(n, \%)$ & $48(3.4 \%)$ & $88(17 \%)$ & $<0.001$ \\
\hline Pregnancy-specific Medications $(n, \%)$ & $67(4.7 \%)$ & $87(16.8 \%)$ & $<0.001$ \\
\hline Tocolytic agent $(n, \%)$ & $32(2.2 \%)$ & $11(2.1 \%)$ & 0.62 \\
\hline Antenatal corticosteroid $(n, \%)$ & $67(4.7 \%)$ & $87(16.8 \%)$ & $<0.001$ \\
\hline
\end{tabular}


Table 1 (continued)

\begin{tabular}{|c|c|c|c|}
\hline Variables & Pre-variant group $(n=1416)$ & $\begin{array}{l}\text { Post-variant group } \\
(n=519)\end{array}$ & $p$ value \\
\hline COVID-19 Therapy $(n, \%)$ & $780(55.1 \%)$ & $355(68.4 \%)$ & $<0.001$ \\
\hline Low-molecular weight heparin $(n, \%)$ & $723(51.1 \%)$ & $360(69.4 \%)$ & $<0.001$ \\
\hline Hydroxychloroquine $(n, \%)$ & $162(11.4 \%)$ & $49(9.4 \%)$ & 0.04 \\
\hline Systemic corticosteroid $(n, \%)$ & $113(7.9 \%)$ & $115(22.4 \%)$ & $<0.001$ \\
\hline Favipiravir $(n, \%)$ & $82(5.8 \%)$ & $50(9.6 \%)$ & 0.03 \\
\hline Lopinavir-ritonavir $(n, \%)$ & $78(5.5 \%)$ & $26(5 \%)$ & 0.26 \\
\hline Convalescent plasma $(n, \%)$ & $37(2.6 \%)$ & $3(0.6 \%)$ & 0.02 \\
\hline Azithromycin $(n, \%)$ & $30(2.1 \%)$ & $29(5.6 \%)$ & 0.001 \\
\hline $\mathrm{N}$-acetylcysteine $(n, \%)$ & $24(1.7 \%)$ & $37(7.1 \%)$ & $<0.001$ \\
\hline rHuIL-1Ra $(n, \%)$ & $20(1.4 \%)$ & $13(2.5 \%)$ & 0.22 \\
\hline Colchicine $(n, \%)$ & $6(0.4 \%)$ & $17(3.3 \%)$ & 0.007 \\
\hline High-dose vitamin $\mathrm{C}(n, \%)$ & $5(0.3 \%)$ & $30(5.8 \%)$ & $<0.001$ \\
\hline Remdesivir $(n, \%)$ & $4(0.3 \%)$ & $2(0.4 \%)$ & 0.53 \\
\hline Tocilizumab $(n, \%)$ & $1(0.07 \%)$ & $2(0.4 \%)$ & 0.16 \\
\hline Dornase alpha $(n, \%)$ & $1(0.07 \%)$ & $1(0.2 \%)$ & 0.92 \\
\hline Antibiotherapy for other pathogens $(n, \%)$ & $280(19.7 \%)$ & $159(30.6 \%)$ & $<0.001$ \\
\hline \multicolumn{4}{|l|}{ COVID-19 severity } \\
\hline Mild $(n, \%)$ & $1322(93.4 \%)$ & $398(77.4 \%)$ & \multirow[t]{4}{*}{$<0.001$} \\
\hline Moderate $(n, \%)$ & $65(4.6 \%)$ & $66(12.8 \%)$ & \\
\hline Severe $(n, \%)$ & $14(1 \%)$ & $22(4.3 \%)$ & \\
\hline Critic $(n, \%)$ & $15(1 \%)$ & $28(5.4 \%)$ & \\
\hline Respiratory support $(n, \%)$ & $103(7.3 \%)$ & $139(26.8 \%)$ & $<0.001$ \\
\hline Nasal oxygen therapy $(n, \%)$ & $76(5.4 \%)$ & $70(13.5 \%)$ & \multirow[t]{4}{*}{$<0.001$} \\
\hline High-flow nasal cannula $(n, \%)$ & $12(0.8 \%)$ & $22(4.2 \%)$ & \\
\hline Oxygen mask with reservoir bag & $8(0.5 \%)$ & $28(5.4 \%)$ & \\
\hline Invasive mechanical ventilation $(n, \%)$ & $7(0.4 \%)$ & $27(5.2 \%)$ & \\
\hline ICU admission $(n, \%)$ & $26(1.8 \%)$ & $67(12.9 \%)$ & $<0.001$ \\
\hline Maternal mortality $(n, \%)$ & $6(0.4 \%)$ & $15(2.9 \%)$ & $<0.001$ \\
\hline Hospitalization rate $(n, \%)$ & $792(55.9 \%)$ & $121(23.3 \%)$ & $<0.001$ \\
\hline Length of hospital stay $(\operatorname{mean} \pm \mathrm{SD})(\min -\max )$ & $3.15 \pm 3.76(1-35)$ & $4.64 \pm 8.21(1-54)$ & 0.03 \\
\hline
\end{tabular}

COVID-19, Coronavirus disease 19; BMI, body-mass index; ICU, intensive care unit; IQR, inter-quartile range; ITP, 1mmune thrombocytopenic purpura; SD, standard deviation; rHuIL-1Ra, recombinant human IL-1 receptor antagonist

cohort study from Italy including 3306 pregnant women with confirmed SARS-CoV-2 infection reported increased rates of oxygen support requirement and ICU admission among cases with pneumonia during the Alpha variant period compared to the wild-type period [14]. A case report from Belgium presented placentitis and acute placental insufficiency in a pregnant woman infected by the Alpha variant [15]. Another single-center retrospective cohort study from the United States compared the pregnancy outcomes between cases admitted to hospital before and after the Delta variant periods. The mentioned study reported that cases diagnosed after the spread of the Delta variant were more symptomatic and their gestational age at diagnosis was earlier [16]. Similarly, a single-center retrospective cohort study from another tertiary center from the United States compared the outcomes of pregnant women diagnosed in the pre-Delta variant period with those diagnosed in the Delta period indicating increased rates of critical illness and adverse perinatal outcomes [17]. A multicenter prospective cohort study from the United States including 1515 pregnant women reported increased COVID-19-related morbidity after the predomination of the Delta variant especially in populations with lower vaccine acceptance [18]. A retrospective observational cohort study from India comparing pregnant and postpartum women according to the first and second waves of SARSCoV-2 infection reported higher rates of severe COVID-19, admission to ICU, and maternal mortality [19]. Finally, correspondence from the United Kingdom underlined the more severe course of COVID-19 for pregnant and peripartum women in the second wave of the pandemic compared to the first wave [20]. The findings of the present study were consistent with the current literature. However, to the best of our 
Table 2 Comparison of initial laboratory test results and radiologic imaging findings between the pre-variant and post-variant groups

\begin{tabular}{|c|c|c|c|}
\hline Variables & Pre-variant group $(n=1416)$ & $\begin{array}{l}\text { Post-variant group } \\
(n=519)\end{array}$ & $p$ value \\
\hline $\mathrm{Hb}(\mathrm{g} / \mathrm{dl})(\operatorname{mean} \pm \mathrm{SD})(\min -\max )$ & $11.88 \pm 1.43(4.7-16.4)$ & $11.82 \pm 1.48(7.5-16)$ & 0.22 \\
\hline $\operatorname{Hct}(\%)(\operatorname{mean} \pm \mathrm{SD})(\min -\max )$ & $35.98 \pm 4.27(26.2-47.1)$ & $35.96 \pm 6.48(23.4-46.2)$ & 0.89 \\
\hline $\mathrm{Hb}<10 \mathrm{~g} / \mathrm{dl}(n, \%)$ & $137(9.7 \%)$ & $53(10.2 \%)$ & 0.43 \\
\hline Leukocyte $\left(10^{3} / \mathrm{mm}^{3}\right)($ mean $\pm \mathrm{SD})(\min -\max )$ & $7412.40 \pm 2889.65(1240-28,510)$ & $\begin{array}{l}8494.32 \pm 3648.56 \\
(2750-25,180)\end{array}$ & $<0.001$ \\
\hline Leukocytosis $\left(>11,000 / \mathrm{mm}^{3}\right)(n, \%)$ & $145(10.2 \%)$ & $102(19.7 \%)$ & $<0.001$ \\
\hline Neutrophil $\left(10^{3} / \mathrm{mm}^{3}\right)(\operatorname{mean} \pm \mathrm{SD})(\min -\max )$ & $5476.82 \pm 2629.86(1070-25,430)$ & $\begin{array}{l}6541.19 \pm 3253.63 \\
(990-23,890)\end{array}$ & $<0.001$ \\
\hline Neutrophil percentage $(\%)($ mean $\pm S D)(\min -\max )$ & $75.74 \pm 10.84(62-90.8)$ & $76.03 \pm 9.20(35.5-94.9)$ & 0.94 \\
\hline Neutrophilia $\left(>7700 / \mathrm{mm}^{3}\right.$ or $>70 \%$ of leukocytes) $(n, \%)$ & $218(15.4 \%)$ & $136(26.2 \%)$ & $<0.001$ \\
\hline Lymphocyte $\left(10^{3} / \mathrm{mm}^{3}\right)($ mean $\pm \mathrm{SD})(\min -\max )$ & $\begin{array}{l}1330.67 \pm 576.97 \\
(140-4650)\end{array}$ & $\begin{array}{l}1094.37 \pm 467.24 \\
(100-2970)\end{array}$ & $<0.001$ \\
\hline Lymphocyte percentage $(\%)(\operatorname{mean} \pm \mathrm{SD})(\min -\max )$ & $22.40 \pm 9.51(1.8-51.8)$ & $13.38 \pm 8.75(1.7-53)$ & 0.002 \\
\hline Lymphocytopenia $\left(<1000 / \mathrm{mm}^{3}\right.$ or $<8 \%$ of leukocytes $)(n, \%)$ & $433(30.6 \%)$ & $271(52.2 \%)$ & $<0.001$ \\
\hline Neutrophil to lymphocyte ratio (mean \pm SD) (min-max) & $5.51 \pm 3.39(1.2-25.28)$ & $6.32 \pm 4.72(1-51.9)$ & 0.42 \\
\hline Platelet $\left(10^{3} / \mathrm{mm}^{3}\right)(\operatorname{mean} \pm \mathrm{SD})(\min -\max )$ & $\begin{array}{l}225.69 \pm 68.55 \\
(19-708)\end{array}$ & $\begin{array}{l}227.32 \pm 101.48 \\
(28-778)\end{array}$ & 0.71 \\
\hline $\operatorname{ESR}(\mathrm{mm} / \mathrm{h})(\operatorname{mean} \pm \mathrm{SD})(\min -\max )$ & $41.10 \pm 23.64(2-113)$ & $47.76 \pm 22.58(3-99)$ & 0.23 \\
\hline $\mathrm{CRP}(\mathrm{mg} / \mathrm{dl})(\operatorname{mean} \pm \mathrm{SD})(\min -\max )$ & $22.78 \pm 32.76(1-419)$ & $34.14 \pm 42.06(2-264)$ & $<0.001$ \\
\hline Procalcitonin $(\mathrm{ng} / \mathrm{ml})(\operatorname{mean} \pm \mathrm{SD})(\min -\max )$ & $0.16 \pm 2.87(0-96.85)$ & $0.24 \pm 1.72(0-28.63)$ & 0.58 \\
\hline IL-6 $(\mathrm{pg} / \mathrm{ml})(\operatorname{mean} \pm \mathrm{SD})(\min -\max )$ & $\begin{array}{l}73.15 \pm 134.19 \\
(0-22,524)\end{array}$ & $\begin{array}{l}176.74 \pm 86.97 \\
(0-5500)\end{array}$ & 0.51 \\
\hline Ferritin $(\mathrm{ng} / \mathrm{ml})($ mean $\pm \mathrm{SD})(\min -\max )$ & $\begin{array}{l}50.04 \pm 269.21 \\
(10-9130)\end{array}$ & $\begin{array}{l}99.78 \pm 354.60 \\
(2-6263)\end{array}$ & 0.004 \\
\hline $\mathrm{BUN}(\mathrm{mmol} / \mathrm{L})(\operatorname{mean} \pm \mathrm{SD})(\min -\max )$ & $16.68 \pm 8.13(9-75)$ & $17.28 \pm 14.59(8-74)$ & 0.38 \\
\hline Creatinine $(\mathrm{mg} / \mathrm{dl})(\operatorname{mean} \pm \mathrm{SD})(\min -\max )$ & $0.57 \pm 2.44(0.30-4.85)$ & $0.51 \pm 0.34(0.40-6.81)$ & 0.54 \\
\hline $\operatorname{ALT}(\mathrm{IU} / \mathrm{L})(\operatorname{mean} \pm \mathrm{SD})(\min -\max )$ & $\begin{array}{l}26.12 \pm 41.73 \\
(8-884)\end{array}$ & $\begin{array}{l}43.21 \pm 104.42 \\
(10-1761)\end{array}$ & $<0.001$ \\
\hline $\mathrm{AST}(\mathrm{IU} / \mathrm{L})(\operatorname{mean} \pm \mathrm{SD})(\min -\max )$ & $28.10 \pm 45.80(9-1065)$ & $51.59 \pm 138.22(6-1783)$ & $<0.001$ \\
\hline $\begin{array}{l}\text { Elevated liver enzymes (n, \%) } \\
\text { (ALT and AST } \geq \text { twice the upper limit) }\end{array}$ & $66(4.6 \%)$ & $126(24.2 \%)$ & $<0.001$ \\
\hline LDH (IU/L) (mean \pm SD) (min-max) & $\begin{array}{l}220.66 \pm 138.98 \\
(125-3780)\end{array}$ & $\begin{array}{l}291.41 \pm 285.28 \\
(120-5730)\end{array}$ & $<0.001$ \\
\hline D-Dimer $(\mathrm{mcg} / \mathrm{mL})(\min -\max )$ & $2.08 \pm 3.26(0.01-67.25)$ & $2.61 \pm 4.33(0.20-35.21)$ & 0.01 \\
\hline CK-MB $(\mathrm{ng} / \mathrm{ml})(\operatorname{mean} \pm \mathrm{SD})(\min -\max )$ & $1.10 \pm 2.32(0-45.6)$ & $1.13 \pm 2.06(0-19.4)$ & 0.78 \\
\hline Troponin $(\mathrm{ng} / \mathrm{ml})(\operatorname{mean} \pm \mathrm{SD})(\min -\max )$ & $5.30 \pm 77.85(0-654)$ & $18.17 \pm 137.69(0-2024)$ & 0.03 \\
\hline Hypokalemia $(\mathrm{K}<2.5 \mathrm{mmol} / \mathrm{L})(n, \%)$ & $41(2.9 \%)$ & $35(6.7 \%)$ & 0.06 \\
\hline Radiologic imaging $(n, \%)$ & $167(11.8 \%)$ & $76(14.6 \%)$ & 0.52 \\
\hline Chest X-ray $(n, \%)$ & $103(7.3 \%)$ & $68(13.1 \%)$ & 0.24 \\
\hline Chest CT $(n, \%)$ & $80(5.6 \%)$ & $26(5 \%)$ & 0.72 \\
\hline Pulmoner CT angiography $(n, \%)$ & $6(0.4 \%)$ & $8(1.5 \%)$ & 0.003 \\
\hline Radiologic imaging findings suspicious for COVID-19 $(n, \%)$ & $111(7.8 \%)$ & $85(16.4 \%)$ & 0.42 \\
\hline
\end{tabular}

ALT, alanine aminotransferase; AST, aspartate aminotransferase; BUN, blood urea nitrogen; COVID-19, Coronavirus disease 19; CRP, C-reactive protein; ESR, erythrocyte sedimentation rate; Hb, hemoglobin; Hct, hematocrit; IL-6, Interleukin 6; IQR, inter-quartile range; LDH, lactate dehydrogenase; SD, standard deviation

knowledge, the present study was the most comprehensive study from a tertiary reference center dealing with pregnant women with COVID-19. In our opinion, the experience of Ankara City Hospital may guide health care systems to establish more effective clinical management protocols.

Pregnancy is a unique period accompanied by many physiological, endocrine, and immunological events [34, 
Table 3 Comparison of obstetric and neonatal outcomes between the pre-variant and post-variant groups

\begin{tabular}{|c|c|c|c|}
\hline Variables & Pre-variant group $(n=1416)$ & $\begin{array}{l}\text { Post-variant group } \\
(n=519)\end{array}$ & $p$ value \\
\hline Pregnancy complications $(n, \%)$ & $267(18.8 \%)$ & $237(45.6 \%)$ & 0.007 \\
\hline Threatened abortion $(n, \%)$ & $10(0.7 \%)$ & $8(1.5 \%)$ & 0.23 \\
\hline Miscarriage $(n, \%)$ & $34(2.4 \%)$ & $20(3.8 \%)$ & 0.02 \\
\hline Hiperemezis Gravidarum $(n, \%)$ & $10(0.7 \%)$ & $5(0.9 \%)$ & 0.54 \\
\hline Cholestasis of pregnancy $(n, \%)$ & $15(1 \%)$ & $11(2.1 \%)$ & 0.03 \\
\hline Fetal anomaly $(n, \%)^{*}$ & $17(1.2 \%)$ & $2(0.3 \%)$ & 0.12 \\
\hline Intrauterine fetal demise $(n, \%)$ & $24(1.7 \%)$ & $11(2.1 \%)$ & 0.53 \\
\hline Fetal growth restriction $(n, \%)$ & $18(1.2 \%)$ & $6(1.2 \%)$ & 0.62 \\
\hline Gestational diabetes $(n, \%)$ & $24(1.7 \%)$ & $13(2.5 \%)$ & 0.09 \\
\hline Gestational hypertension $(n, \%)$ & $13(0.9 \%)$ & $7(1.3 \%)$ & 0.13 \\
\hline Preterm labor $(n, \%)$ & $62(4.4 \%)$ & $137(26.4 \%)$ & $<0.001$ \\
\hline Preterm premature rupture of the membranes $(n, \%)$ & $16(1.1 \%)$ & $8(1.5 \%)$ & 0.24 \\
\hline Preeclampsia $(n, \%)$ & $13(0.9 \%)$ & $6(1.1 \%)$ & 0.32 \\
\hline $\operatorname{Eclampsia}(n, \%)$ & $2(0.1 \%)$ & $1(0.1 \%)$ & 0.89 \\
\hline Placental abruption $(n, \%)$ & $3(0.2 \%)$ & $8(1.5 \%)$ & $<0.001$ \\
\hline Deep vein thrombosis $(n, \%)$ & $3(0.2 \%)$ & $1(0.1 \%)$ & 0.75 \\
\hline Clavicle fracture $(n, \%)$ & $3(0.2 \%)$ & $1(0.1 \%)$ & 0.83 \\
\hline \multicolumn{4}{|l|}{ Delivery status } \\
\hline Pregnancy loss $(n, \%)$ & $58(4.1 \%)$ & $33(6.4 \%)$ & $<0.001$ \\
\hline Ongoing pregnancy $(n, \%)$ & $154(10.9 \%)$ & $291(56.1 \%)$ & \\
\hline Delivered $(n, \%)$ & $1204(85 \%)$ & $195(37.5 \%)$ & \\
\hline $\begin{array}{l}\text { The time interval between diagnosis and delivery (days) (mean } \pm \text { SD) } \\
(\text { min-max) }\end{array}$ & $7.82 \pm 9.12(1-34)$ & $11.49 \pm 10.54(1-60)$ & 0.23 \\
\hline \multicolumn{4}{|l|}{ Route of delivery } \\
\hline Normal spontaneous vaginal delivery $(n, \%)$ & $509(42.3 \%)$ & $77(39.5 \%)$ & 0.64 \\
\hline Cesarean section $(n, \%)$ & $692(57.4 \%)$ & $117(60 \%)$ & \\
\hline Vaginal birth after cesarean section $(n, \%)$ & $3(0.3 \%)$ & $1(0.5 \%)$ & \\
\hline \multicolumn{4}{|l|}{ Cesarean indications } \\
\hline Previous cesarean section $(n, \%)$ & $276(39.8 \%)$ & $37(31.6 \%)$ & 0.08 \\
\hline Fetal distress $(n, \%)$ & $198(28.6 \%)$ & $31(26.5 \%)$ & 0.63 \\
\hline Cefalopelvic dysproportion $(n, \%)$ & $124(17.9 \%)$ & $8(6.8 \%)$ & 0.02 \\
\hline Maternal health condition $(n, \%)$ & $36(5.2 \%)$ & $28(23.9 \%)$ & $<0.001$ \\
\hline Malpresentation $(n, \%)$ & $30(4.3 \%)$ & $9(7.7 \%)$ & 0.11 \\
\hline Multiple pregnancy $(n, \%)$ & $16(2.3 \%)$ & $2(1.7 \%)$ & 0.68 \\
\hline $\operatorname{Macrosomia}(n, \%)$ & $12(1.7 \%)$ & $2(1.7 \%)$ & 0.98 \\
\hline \multicolumn{4}{|l|}{ Labor anesthesia } \\
\hline None $(n, \%)$ & $506(42.1 \%)$ & $75(38.4 \%)$ & $<0.001$ \\
\hline General $(n, \%)$ & $36(2.9 \%)$ & $20(10.2 \%)$ & \\
\hline Regional $(n, \%)$ & $662(54.9 .9 \%)$ & $100(51.3 \%)$ & \\
\hline Spontaneous labor $(n, \%)$ & $1021(84.8 \%)$ & $153(78.4 \%)$ & 0.02 \\
\hline Preterm delivery (n, \%) & $228(18.9 \%)$ & $87(44.6 \%)$ & $<0.001$ \\
\hline Gestational age at delivery (weeks) $($ mean \pm SD) $(\min -\max )$ & $37.63 \pm 2.43(26-42)$ & $35.53 \pm 4.70(25-41)$ & 0.03 \\
\hline Birth weight $(\mathrm{g})(\operatorname{mean} \pm \mathrm{SD})(\min -\max )$ & $\begin{array}{l}3141.15 \pm 617.42 \\
(540-4550)\end{array}$ & $\begin{array}{l}2671 \pm 920.52 \\
(560-4195)\end{array}$ & $<0.001$ \\
\hline Apgar 1st minute (median) (IQR, min-max) & $8(1,6-9)$ & $7(2,6-9)$ & 0.01 \\
\hline Apgar 5th minute (median) (IQR, min-max) & $9(1,8-10)$ & $9(1,8-10)$ & 0.23 \\
\hline NICU admission $(n, \%)$ & $230(18.8 \%)$ & $67(34 \%)$ & $<0.001$ \\
\hline Neonatal SARS-CoV-2 positivity $(n, \%)$ & $1(0.2 \%)$ & $0(0 \%)$ & 0.76 \\
\hline SARS-CoV-2 positivity in the breastmilk & $1(0.2 \%)$ & $0(0 \%)$ & 0.85 \\
\hline
\end{tabular}

BMI, body-mass index. COVID-19, Coronavirus disease 19. IQR, inter-quartile range; NICU, neonatal intensive care unit; RT-PCR, real-time polymerase chain reaction; SARS-CoV-2, severe acute respiratory syndrome coronavirus 2; SD, standard deviation 
Table 4 Correlation of the post-variant period with disease severity and maternal mortality

\begin{tabular}{|c|c|c|c|c|}
\hline & \multicolumn{2}{|c|}{ Disease severity } & \multicolumn{2}{|c|}{ Maternal mortality } \\
\hline & $r$ & $p$ & $r$ & $p$ \\
\hline $\begin{array}{l}\text { The post-variant } \\
\text { period }\end{array}$ & 0.19 & $<0.001$ & 0.12 & $<0.001$ \\
\hline
\end{tabular}

35]. The aforementioned changes make the pregnant population vulnerable to some infectious agents [36-38]. Therefore, physicians dealing with pregnant women should be cautious in the management of infectious diseases in this specific population. As SARS-CoV-2 was also reported to have a more severe course in pregnant patients, follow-up and treatment of these cases should be performed by an experienced multidisciplinary team [23]. The most important reason for planning the current study was the significant increase observed in intensive care unit admission, obstetric complications, and maternal mortality in pregnant patients with COVID-19 after the variant period. Although the experience on COVID-19 has increased significantly, it is, unfortunately, difficult to prevent the negative effects of the disease on pregnant women. Thus, encouraging pregnant women for COVID19 vaccination, early diagnosis of disease, meticulous follow-up, multidisciplinary approach, and administration of appropriate medications seem to be the main strategies for preventing adverse outcomes [23, 39, 40].

Readers may be concerned about a selection bias due to the design of the current study. As our experience and knowledge have increased on SARS-CoV-2, management protocols have been updated regularly. Moreover, attitudes of patients have been deeply affected from the cumulative knowledge. Thus, comparing two different periods based on parameters like hospitalization, treatment choices and delivery characteristics may be misguiding. However, the significantly increasing rates of ICU admission, maternal mortality and pregnancy complications in the post-variant period are indicative for a worse course of SARS-CoV-2 after the variants in pregnant population.

The main strengths of the present study were prospective design, the inclusion of comprehensive study parameters, and large number of cases. On the other hand, singlecenter experience and lack of information related to the long-term outcomes were the main limitations.

In conclusion, the post-variant COVID-19 period was associated with a severe course of the disease and increased rates of adverse obstetric outcomes in pregnant patients.

Acknowledgements Special thanks to all the health care staff of our hospital who work devotedly for the health of our community during the pandemic period.
Author contributions All the authors cited in the manuscript had substantial contributions to the concept and design, the execution of the work, or the analysis and interpretation of data; drafting or revising the manuscript, and have read and approved the final version of the paper. DS: conceptualization, methodology, visualization, reviewing and editing. AT: original draft preparation, writing, data collection. ATA: data collection, writing. SS: data collection, writing. BB: data collection, writing. DO: data collection, writing. DUH: data collection, writing. DMB reviewing and editing. MY: literature search. BS: reviewing and editing. SAE: data collection, writing. YC: data collection, writing. SGA: data collection, writing. ET: data collection, writing. SU: data collection, writing. FEC: data collection, writing. SI: resources, analysis/interpretation. ST: analysis/interpretation. AAS: supervision. OMT: project development.

Funding No funding was used for this study.

\section{Declarations}

Conflict of interest The authors state that they have no conflict of interest in this study.

\section{References}

1. Tull MT, Edmonds KA, Scamaldo KM, Richmond JR, Rose JP, Gratz KL (2020) Psychological outcomes associated with stay-athome orders and the perceived impact of COVID-19 on daily life. Psychiatry Res 289:113098

2. Singh S, Nair VG, Singh VV, Tiwari S, Arora D, Dey M, Tiwari R, Nair SV (2021) Pregnancy-specific concerns and psychological impact of COVID-19 on antenatal women. Gynecol Obstetr Reprod Med 2021:1-6. https://doi.org/10.21613/GORM.2021. 1172

3. Papoutsi E, Giannakoulis VG, Ntella V, Pappa S, Katsaounou P (2020) Global burden of COVID-19 pandemic on healthcare workers. Eur Respiratory Soc 6:2

4. Galli F, Pozzi G, Ruggiero F, Mameli F, Cavicchioli M, Barbieri S, Canevini MP, Priori A, Pravettoni G, Sani G (2020) A systematic review and provisional metanalysis on psychopathologic burden on health care workers of coronavirus outbreaks. Front Psych 2020:11

5. Saha S, Tanmoy AM, Tanni AA, Goswami S, Al Sium SM, Saha S, Islam S, Hooda Y, Malaker AR, Anik AM (2021) New waves, new variants, old inequity: a continuing COVID-19 crisis. BMJ Glob Health 6(8):e007031

6. Zhao Y, Huang J, Zhang L, Chen S, Gao J, Jiao H (2021) The global transmission of new coronavirus variants. Environ Res 2021:112240

7. Villar J, Ariff S, Gunier RB, Thiruvengadam R, Rauch S, Kholin A, Roggero P, Prefumo F, Do Vale MS, Cardona-Perez JA (2021) Maternal and neonatal morbidity and mortality among pregnant women with and without COVID-19 infection: the INTERCOVID multinational cohort study. JAMA Pediatr 175:817

8. Zambrano LD, Ellington S, Strid P, Galang RR, Oduyebo T, Tong VT, Woodworth KR, Nahabedian JF 3rd, Azziz-Baumgartner E, Gilboa SM, Meaney-Delman D (2020) Update: characteristics of symptomatic women of reproductive age with laboratoryconfirmed SARS-CoV-2 infection by pregnancy status-United States, January 22-October 3. MMWR Morb Mortal Wkly Rep 69(44):1641-1647. https://doi.org/10.15585/mmwr.mm6944e3

9. Martinez-Portilla RJ, Sotiriadis A, Chatzakis C, Torres-Torres J, Espino YSS, Sandoval-Mandujano K, Castro-Bernabe DA, Medina-Jimenez V, Monarrez-Martin JC, Figueras F, Poon LC 
(2021) Pregnant women with SARS-CoV-2 infection are at higher risk of death and pneumonia: propensity score matched analysis of a nationwide prospective cohort (COV19Mx). Ultrasound Obstetr Gynecol 57(2):224-231. https://doi.org/10.1002/uog.23575

10. Dey M, Singh S, Tiwari R, Nair VG, Arora D, Tiwari S (2021) Pregnancy Outcome in first 50 SARS-Cov-2 positive patients at our center. Gynecol Obstetr Reprod Med 27(1):11-16. https://doi. org/10.21613/GORM.2021.1176

11. Mirbeyk M, Saghazadeh A, Rezaei N (2021) A systematic review of pregnant women with COVID-19 and their neonates. Arch Gynecol Obstet 304(1):5-38. https://doi.org/10.1007/ s00404-021-06049-z

12. Jafari M, Pormohammad A, Sheikh Neshin SA, Ghorbani S, Bose D, Alimohammadi S, Basirjafari S, Mohammadi M, RasmussenIvey C, Razizadeh MH (2021) Clinical characteristics and outcomes of pregnant women with COVID-19 and comparison with control patients: a systematic review and meta-analysis. Rev Med Virol 31:e2208

13. Akhtar H, Patel C, Abuelgasim E, Harky A (2020) COVID-19 (SARS-CoV-2) infection in pregnancy: a systematic review. Gynecol Obstet Invest 85(4):295-306

14. Donati S, Corsi E, Maraschini A, Salvatore MA (2021) SARSCoV-2 infection among hospitalized pregnant women and impact of different viral strains on COVID-19 severity in Italy: a national prospective population-based cohort study. BJOG. https://doi.org/ 10.1111/1471-0528.16980

15. Dumont S, Balduyck J, Reynders M, Vanwalleghem L, Lebbe B (2021) Acute SARS-CoV-2 alpha variant infection leading to placental insufficiency and fetal distress. J Med Virol. https://doi. org/10.1002/jmv. 27379

16. Wang AM, Berry M, Moutos CP, Omere C, Clark SM, Harirah HM, Jain S, Olson GL, Pacheco LD, Saade GR, Saad AF (2021) Association of the Delta (B.1.617.2) variant of severe acute respiratory syndrome coronavirus 2 (SARS-CoV-2) with pregnancy outcomes. Obstetr Gynecol. https://doi.org/10.1097/aog.00000 00000004595

17. Seasely AR, Blanchard CT, Arora N, Battarbee AN, Casey BM, Dionne-Odom J, Sinkey RG, Szychowski JM, Tita AT, Subramaniam A (2021) Maternal and perinatal outcomes associated with the severe acute respiratory syndrome coronavirus 2 (SARSCoV-2) delta (B.1.617.2) Variant. Obstetr Gynecol. https://doi. org/10.1097/aog.0000000000004607

18. Adhikari EH, SoRelle JA, McIntire DD, Spong CY (2021) Increasing severity of COVID-19 in pregnancy with Delta (B.1.617.2) variant surge. Am J Obstet Gynecol. https://doi.org/ 10.1016/j.ajog.2021.09.008

19. Mahajan NN, Pophalkar M, Patil S, Yewale B, Chaaithanya IK, Mahale SD, Gajbhiye RK (2021) Pregnancy outcomes and maternal complications during the second wave of coronavirus disease 2019 (covid-19) in India. Obstet Gynecol 138(4):660

20. Kadiwar S, Smith JJ, Ledot S, Johnson M, Bianchi P, Singh N, Montanaro C, Gatzoulis M, Shah N, Ukor E-F (2021) Were pregnant women more affected by COVID-19 in the second wave of the pandemic? The Lancet 397(10284):1539-1540

21. Moltner S, de Vrijer B, Banner H (2021) Placental infarction and intrauterine growth restriction following SARS-CoV-2 infection. Arch Gynecol Obstet 304(6):1621-1622. https://doi.org/10.1007/ s00404-021-06176-7

22. Di Guardo F, Di Grazia FM, Di Gregorio LM, Zambrotta E, Carrara G, Gulino FA, Tuscano A, Palumbo M (2021) Poor maternalneonatal outcomes in pregnant patients with confirmed SARSCov-2 infection: analysis of 145 cases. Arch Gynecol Obstet 303(6):1483-1488. https://doi.org/10.1007/s00404-020-05909-4

23. Sahin D, Tanacan A, Erol SA, Yucel Yetiskin FD, Besimoglu B, Ozden Tokalioglu E, Anuk AT, Turgut E, Goncu Ayhan S, Turgay B, Unlu S, Kanmaz G, Dinc B, Ozgu-Erdinc AS, Keskin HL,
Surel AA, Moraloglu Tekin O (2021) Management of pregnant women with COVID-19: a tertiary pandemic center experience on 1416 cases. J Med Virol. https://doi.org/10.1002/jmv.27423

24. Sahin D, Tanacan A, Erol SA, Anuk AT, Yetiskin FDY, Keskin HL, Ozcan N, Ozgu-Erdinc AS, Eyi EGY, Yucel A, Tayman C, Unlu S, Dinc B, Sari E, Surel AA, Moraloglu OT (2021) Updated experience of a tertiary pandemic center on 533 pregnant women with COVID-19 infection: A prospective cohort study from Turkey. Int J Gynaecol Obstetr 152(3):328-334. https://doi.org/10. 1002/ijgo. 13460

25. Sahin D, Tanacan A, Erol SA, Anuk AT, Eyi EGY, Ozgu-Erdinc AS, Yucel A, Keskin HL, Tayman C, Unlu S, Kirca F, Dinc B, San I, Parpucu ÜM, Surel AA, Moraloglu OT (2020) A pandemic center's experience of managing pregnant women with COVID19 infection in Turkey: A prospective cohort study. Int journal of gynaecology and obstetrics: the official organ of the International Federation of Gynaecology and Obstetrics 151(1):74-82. https:// doi.org/10.1002/ijgo.13318

26. RCOG Coronavirus (COVID-19) Infection in Pregnancy Information for healthcare professionals. https://www.rcog.org.uk/globa lassets/documents/guidelines/2021-02-19-coronavirus-covid-19infection-in-pregnancy-v13.pdf. Accessed 1.5.2021

27. Novel Coronavirus (2019-nCoV) technical guidance. (2020). https://www.who.int/emergencies/diseases/novel-coronavirus2019/technical-guidance Accessed (Accessed on April 12, 2020)

28. ACOG Novel Coronavirus 2019 (COVID-19). https://www.acog. org/clinical/clinical-guidance/practice-advisory/articles/2020/03/ novel-coronavirus-2019. Accessed 1.5.2021

29. Turkish Ministry of Health, General Directorate of Public Health, COVID-19 (SARS-CoV-2 infection) Guideline, Scientific Committee Report. https://covid19bilgi.saglik.gov.tr/depo/rehberler/ COVID-19_Rehberi.pdf?type=file. Accessed 21.08.2020

30. Abdool Karim SS, de Oliveira T (2021) New SARS-CoV-2 variants-clinical, public health, and vaccine implications. N Engl J Med 384(19): 1866-1868

31. Walensky RP, Walke HT, Fauci AS (2021) SARS-CoV-2 variants of concern in the United States-Challenges and opportunities. JAMA 325(11):1037-1038

32. Fontanet A, Autran B, Lina B, Kieny MP, Karim SSA, Sridhar D (2021) SARS-CoV-2 variants and ending the COVID-19 pandemic. The Lancet 397(10278):952-954

33. Ferrer-Oliveras R, Mendoza M, Capote S, Pratcorona L, EsteveValverde E, Cabero-Roura L, Alijotas-Reig J (2021) Immunological and physiopathological approach of COVID-19 in pregnancy. Arch Gynecol Obstet 304(1):39-57. https://doi.org/10.1007/ s00404-021-06061-3

34. Warning JC, McCracken SA, Morris JM (2011) A balancing act: mechanisms by which the fetus avoids rejection by the maternal immune system. Reproduction 141(6):715-724

35. Morelli SS, Mandal M, Goldsmith LT, Kashani BN, Ponzio NM (2015) The maternal immune system during pregnancy and its influence on fetal development. Research and Reports in Biology 6:171-189

36. Galang RR, Chang K, Strid P, Snead MC, Woodworth KR, House LD, Perez M, Barfield WD, Meaney-Delman D, Jamieson DJ (2020) Severe coronavirus infections in pregnancy: a systematic review. Obstet Gynecol 136(2):262-272

37. Wang R, Yan W, Du M, Tao L, Liu J (2021) The effect of influenza virus infection on pregnancy outcomes: A systematic review and meta-analysis of cohort studies. International Journal of Infectious Diseases

38. Mohr-Sasson A, Chayo J, Bart Y, Meyer R, Sivan E, MazakiTovi S, Yinon Y (2020) Laboratory characteristics of pregnant compared to non-pregnant women infected with SARS-CoV-2. Arch Gynecol Obstet 302(3):629-634. https://doi.org/10.1007/ s00404-020-05655-7 
39. Goncu Ayhan S, Oluklu D, Atalay A, Menekse Beser D, Tanacan A, Moraloglu Tekin O, Sahin D (2021) COVID-19 vaccine acceptance in pregnant women. International journal of gynaecology and obstetrics: the official organ of the International Federation of Gynaecology and Obstetrics. https://doi.org/10.1002/ijgo. 13713

40. Rottenstreich A, Tsur A, Braverman N, Kabiri D, Porat S, Benenson S, Oster Y, Kam HA, Walfisch A, Bart Y, Meyer R, Lifshitz SJ, Amikam U, Biron-Shental T, Cohen G, Sciaky-Tamir Y, Shachar IB, Yinon Y, Reubinoff B (2021) Vaginal delivery in
SARS-CoV-2-infected pregnant women in Israel: a multicenter prospective analysis. Arch Gynecol Obstet 303(6):1401-1405. https://doi.org/10.1007/s00404-020-05854-2

Publisher's Note Springer Nature remains neutral with regard to jurisdictional claims in published maps and institutional affiliations. 